\title{
16-User OFDM-CDMA Optical Access Network
}

\author{
X. Guo ${ }^{1}$, Q. Wang ${ }^{1,3}$, L. Zhou ${ }^{2}$, L. Fang ${ }^{2}$, X, Li ${ }^{1}$, A. Wonfor ${ }^{1}$, R. V. Penty ${ }^{1}$, I. H. White ${ }^{1}$ \\ 1- Centre for Photonic Systems, Department of Engineering, University of Cambridge, 9 J. J. Thomson Avenue, Cambridge, CB3 OFA, UK \\ 2- Huawei Technologies, Bantian, Longgang District, Shenzhen 518129, China \\ 3- Department of Electronic Engineering, Tsinghua University, Beijing 100084, China \\ Email:xg218@cam.ac.uk
}

\begin{abstract}
We demonstrate a $16 \times 2.5 \mathrm{~Gb} / \mathrm{s}$ (40 Gb/s aggregate) OFDM-CDMA PON for nextgeneration access applications. Four-channel error-free transmission over $25 \mathrm{~km}$ SMF shows $6 \mathrm{~dB}$ coding gain, with $0.1 \mathrm{~dB}$ dispersion and $0.9 \mathrm{~dB}$ crosstalk penalties.

OCIS codes: (060.4250) Networks; (060.4510) Optical communications; (060.4080) Modulation.
\end{abstract}

1. Introduction

The exponential growth of global communications nowadays demands optical access networks with high spectral efficiency and high data rates, which also address the scalable and flexible bandwidth allocation issues enabling multiple services. Next Generation Passive Optical Networks (NG-PONs) are forecast to be well beyond $40 \mathrm{~Gb} / \mathrm{s}$ downlink and $10 \mathrm{~Gb} / \mathrm{s}$ uplink rates [1]. However the computational requirement involved in electronic dispersion compensation for traditional modulation schemes becomes more complex and power hungry as the data rate increases. Orthogonal frequency division multiplexing (OFDM) based PONs are now regarded as promising solutions for NG-PONs owing to their high spectral efficiency, good chromatic and polarization mode dispersion tolerance, flexible and dynamic bandwidth allocation capability, as well as the ability to adapt to frequency dependent channel quality with simple single-tap equalization [2]. Various OFDM PON configurations, i.e. time division multiplexing (TDM) OFDM PONs [3] and wavelength division multiplexing (WDM) OFDM PONs [4] have been investigated recently. TDM-OFDM PONs have impairments at higher transmission speeds caused by burst synchronization, interference between different ONUs and upstream beat noise. WDM-OFDM PONs can support high data rates but are relatively expensive and complex due to the use of arrayed waveguide gratings and multiple high speed transceivers. Code division multiple access (CDMA) technologies however have many attractive features, such as single wavelength operation, high power budget margins, a secure physical layer, low interference between users and low optical beat noise. They also allow networking functions such as service monitoring, multicasting and switching [5]. Hence the combination of OFDM and CDMA may lead to a potential evolution route for NG-PONs. Although similar concepts are feasible [6], we feature a different implementation, allowing an improved performance in data rate and demonstrating the feasibility of a higher number of users.

\section{OFDM-CDMA PON Architecture and Experimental Setup}

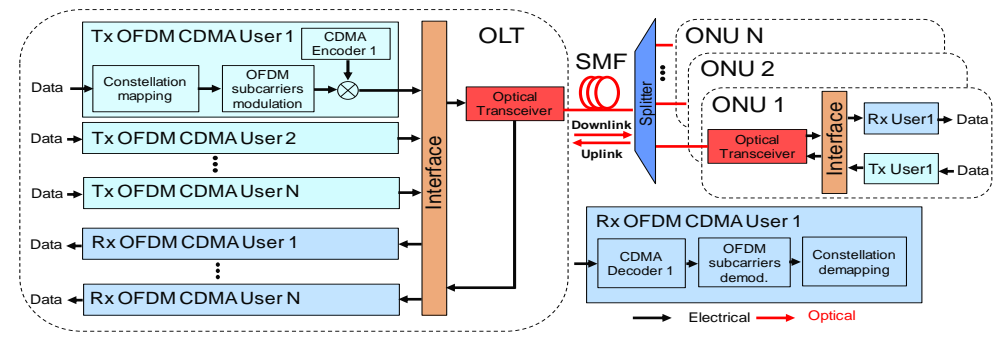

Fig. 1: The proposed OFDM-CDMA PON architecture

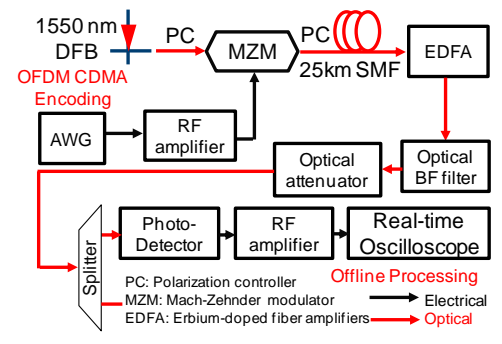

Fig. 2: OFDM-CDMA PON experimental setup

Fig. 1 shows the proposed OFDM-CDMA PON architecture. In the downlink direction, the optical line terminal (OLT) generates unique encoded OFDM data streams for different users which are then simultaneously transmitted to the ONUs. Each user has a corresponding unique CDMA modem which decodes its own data, while rejecting interference from other users, and then demodulates it in an OFDM modem. A similar process applies for the uplink. Fig. 2 shows the OFDM-CDMA PON experimental setup. PRBS bits are mapped to Quadrature Amplitude Modulation (QAM) symbols and then input onto the OFDM subcarriers via a 512-size IFFT. 510 subcarriers are arranged with Hermitian symmetry for real-valued IFFT output suitable for direct detection, although at the cost of lower spectral efficiency. A cyclic prefix of $3 \%$ is added to mitigate the inter-symbol interference (ISI). The complete signal comprises 340 OFDM frames with 40 training pilot frames for the channel estimation and phase equalization. 256 pseudo-noise bits are inserted for automatic system timing synchronization. Each user is encoded with different 16-bit Walsh-Hadamard codes so ideally 16 users can be transmitted simultaneously. The OFDM- 
CDMA data is generated offline and output by an Arbitrary Waveform Generator (AWG) with a fixed sampling rate of $20 \mathrm{GS} / \mathrm{s}$ throughout all the experiments. This modulates a $1550 \mathrm{~nm}$ DFB laser via a $20 \mathrm{GHz}$ Mach-Zehnder modulator (MZM) and the resulting signal is then transmitted through polarization controller (PC), $25 \mathrm{~km}$ of SMF, amplified by an EDFA, filtered by an optical band pass filter (BF) with bandwidth of $\sim 1 \mathrm{~nm}$, then detected by a 20 $\mathrm{GHz}$ receiver (-15 dBm sensitivity at $10 \mathrm{~Gb} / \mathrm{s} \mathrm{NRZ}$ ) and captured by a real-time oscilloscope for offline processing.

\section{Results and Analysis}

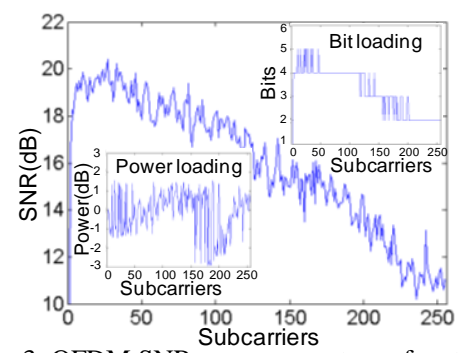

Fig. 3: OFDM SNR measurement as a function of subcarrier number with bit and power loading shown in the insets

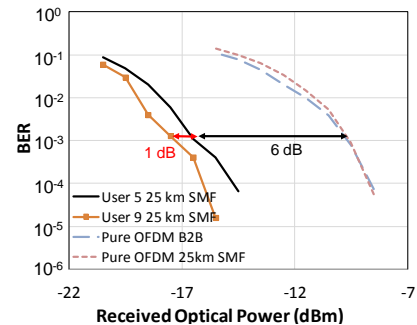

Fig. 4: BER measurements of pure OFDM transmission B2B and after $25 \mathrm{~km}$ of SMF, and 16 QAM OFDM CDMA after $25 \mathrm{~km}$ with single user 5 and user 9

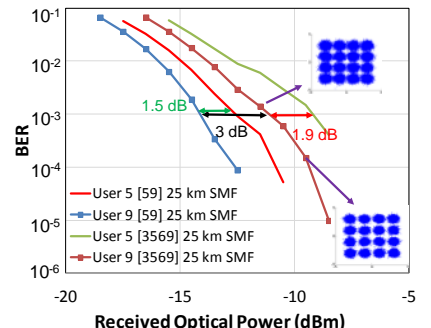

Fig. 5: BER measurements of 16 QAM OFDM CDMA after $25 \mathrm{~km}$ with two and four users (Insets: 16 QAM constellation with BER of $1.4 \times 10^{-3}$ and $1.5 \times 10^{-4}$ )

The channel frequency response over $20 \mathrm{GS} / \mathrm{s}$ sampling rate of different OFDM subcarriers is estimated at an optical power of $-10 \mathrm{dBm}$ as a function of subcarrier number in Fig. 3. The subcarriers occupying high frequencies suffer from larger losses, so adaptive bit and power loading is applied to improve the spectral efficiency. Fig. 4 shows the BER measurements for pure OFDM signals for back-to-back (B2B) operation and after $25 \mathrm{~km}$ of SMF for a spectral efficiency of $1.62 \mathrm{bit} / \mathrm{s} / \mathrm{Hz}$ and a total data rate of $32.4 \mathrm{~Gb} / \mathrm{s}$. There is a negligible $0.1 \mathrm{~dB}$ measured dispersion power penalty. Considering the system noise floor $(-8 \mathrm{dBm})$, the OFDM modulation format is fixed to 16 QAM in the experiment. Fig. 4 also depicts the BER of a 16 QAM OFDM-CDMA PON with different user codes (code 5 and 9) after $25 \mathrm{~km}$ of SMF, demonstrating a $6 \mathrm{~dB}$ coding gain. The BER performance is better than the FEC limit in each case. Fig. 5 shows the BER curves for two and four users, with additional $3 \mathrm{~dB}$ and $6 \mathrm{~dB}$ splitting loss respectively. However when comparing user 5 and user 9 in a one-user system, two-user system and four-user system in Fig. 4 and Fig. 5, there are corresponding increasing penalties of $1 \mathrm{~dB}, 1.5 \mathrm{~dB}$ and $1.9 \mathrm{~dB}$ at a BER of $1 \times 10^{-3}$. This is because different spreading codes have different frequency bandwidths and hence will suffer from different frequency dependent losses, so they have different SNRs even in the one-user system. Also as the user number increases, dispersion and crosstalk degrade the channel orthogonality causing a higher power penalty. Fig. 5 also shows the constellation maps of the received 16 QAM signal at BERs of $1.4 \times 10^{-3}$ and $1.5 \times 10^{-4}$ respectively for the four-user system. The spectral efficiency in this system is $2 \mathrm{bit} / \mathrm{s} / \mathrm{Hz}$ with a single user data rate of $2.5 \mathrm{~Gb} / \mathrm{s}$ shows the viability of $16 \mathrm{ONU}$ channels with an aggregate date rate of $40 \mathrm{~Gb} / \mathrm{s}$ given enough power budget. This could be increased further if more advanced orthogonal codes, higher order modulation formats or higher code chip rates are used. Of course this also requires a more complex synchronization scheme.

\section{Conclusion and Acknowledgement}

We have proposed and demonstrated the feasibility of a novel OFDM-CDMA PON architecture. One, two and four users out of 16-user system have been demonstrated with single channel data rate of $2.5 \mathrm{~Gb} / \mathrm{s}$, potential aggregate data rate $40 \mathrm{~Gb} / \mathrm{s}$. The dispersion penalty is about $0.1 \mathrm{~dB}$ and crosstalk penalty is $0.9 \mathrm{~dB}$ for four users after $25 \mathrm{~km}$ of SMF. The coding gain is $6 \mathrm{~dB}$ compared to the case without CDMA, and this could be improved further using a lower order modulation. We acknowledge Tektronix for supplying arbitrary waveform generator

\section{References}

[1] Y. Luo et al., "Time- and Wavelength-Division Multiplexed Passive Optical Network (TWDM-PON) for Next-Generation PON Stage 2 (NGPON2),” J. Lightwave Technol., Vol. 31, no. 4, p. 587 (2013).

[2] N. Cvijetic et al., "OFDM for Next-Generation Optical Access Networks," J. Lightwave Technol., Vol. 30 no. 4, p. 384 (2012).

[3] H. Yang et al., "DSP-Based Evolution from Conventional TDM-PON to TDM-OFDM-PON," J. Lightwave Technol., Vol. 31 no. 16, p. 2735 (2013).

[4] X. Hu et al., "Energy-efficient WDM-OFDM-PON employing shared OFDM modulation modules in optical line terminal," Opt. Express., Vol. 20, no. 7, p. 8071 (2012).

[5] K. Kitayama, Optical Code Division Multiple Access: A Practical Perspetive, Cambridge University Press, (2014).

[6] L. Zhang et al., "A novel ECDM-OFDM-PON architecture for Next-Generation optical access network,” Opt. Express., Vol. 18, no. 17, p. 18347 (2010). 